Abstract

\title{
New Parameters and Extensive Methodology to Describe the Three Phase Transitions in the $q$-States Clock Model ${ }^{\dagger}$
}

\author{
Oscar Negrete ${ }^{1,2}$, Francisco Peña ${ }^{1}$, Patricio Vargas ${ }^{2,3}$, Eugenio Vogel ${ }^{2,4}$ and Gonzalo Saravia ${ }^{5}$ \\ 1 Departamento de Física, Universidad Técnica Federico Santa María, Valparaíso, Chile \\ 2 Centro para el Desarrollo de la Nanociencia y la Nanotecnología, CEDENNA, Santiago, Chile \\ 3 Department of Physics, Universidad Técnica Federico Santa Maria, Valparaíso, Chile \\ 4 Department of Physics, Universidad de La Frontera, Temuco, Chile \\ 5 Departamento de Ciencias Físicas, Universidad de La Frontera, Temuco, Chile \\ † Presented at the Entropy 2021: The Scientific Tool of the 21st Century, 5-7 May 2021; Available online: \\ https://sciforum.net/conference/Entropy2021/.
}

Published: 5 May 2021

In the $q$-state clock model the spin has q possible orientations in the plane so it can be understood as a generalization of the Ising model for which $q=2$. The Hamiltonian is then the scalar product of the neighboring spins mediated by the ferromagnetic exchange interaction $J$ homogeneous through the square lattice with $L^{\prime} L=N$ spins. It is known that for $q \leq 4$ there is only one phase transition at a temperature $T_{1}$, over which the ferromagnetic phase is lost. Using global order parameters it has been previously established that for $q \geq 5$ this transitions moves steadily to lower temperatures as $q$ increases [1]. For large $L$ the appearing of the so called Berezinskii-Kosterlitz-Thouless (BKT) phase characterized by vortex like structures is established, while a second transition to a disordered phase appears at a higher $T_{2}$ temperature. In the present paper we deeply characterize the nature of this second transition by means of new local order parameters. Surprisingly, an unexpected subtle transition appears at a temperature slightly over the second one (at $\left.T_{3}\right)$ requiring interpretation. This is resolved by considering pure and mixed ferromagnetic, vortex and paramagnetic phases as $T$ increases requiring local order parameters and new methodology to better handle them. Thus, we include now information theory analysis by means of mutability and Shannon entropy characterization. Tendencies towards large $N$ and $q$ values are established.

\section{Reference}

1 Negrete, O.A.; Vargas, P.; Peña, F.J.; Saravia, G.; Vogel, E.E. Entropy and Mutability for the q-State Clock Model in Small Systems. Entropy 2018, 20, 933.

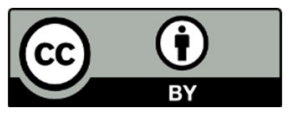

(c) 2021 by the authors. Licensee MDPI, Basel, Switzerland. This article is an open access article distributed under the terms and conditions of the Creative Commons Attribution (CC BY) license (http://creativecommons.org/licenses/by/4.0/). 